\title{
Autoimmune Tissue Scurvy Misdiagnosed as Child Abuse
}

\author{
Michael D Innis \\ MBBS; DTM\&H; FRCPA; FRC Path. Retired Haematologist, Princess Alexandra Hospital, Brisbane, Australia
}

Email address:

micinnis@bigpond.com

\section{To cite this article:}

Michael D Innis. Autoimmune Tissue Scurvy Misdiagnosed as Child Abuse. Clinical Medicine Research. Vol. 2, No. 6, 2013 , pp.154-157.doi: 10.11648/j.cmr.20130206.17

\begin{abstract}
Requests from distressed parents and relatives seeking help after having been falsely accused by doctors of injuring their children are not uncommon. Viraland parasitic infections andvaccinescause an autoimmune disorder, Tissue Scurvy, misdiagnosed as child abuse. This report presents the evidence. Method. Relevant hospital and laboratory reports of three children were examined for evidence of Tissue Scurvy as the cause of the neurological lesions, fractures, bruises and hemorrhages found on them. Results. In all the cases in which appropriate histories and tests were done there was evidence that the doctors either misinterpreted the laboratory evidence or they were unaware of the significance of abnormal tests suggesting Tissue Scurvy as the cause. Conclusion. Some doctors are unaware of the pathophysiological processes of autoimmunity, haemostasis and osteogenesis and are misdiagnosing vaccine induced Tissue Scurvy, absence of Vitamin C within the cell, as Non-accidental Injury.
\end{abstract}

Keywords: Autoimmunity, Tissue Scurvy, Encephalomyelitis, Non-accidental Injury, Shaken Baby Syndrome

\section{Introduction}

At a conference on Encephalitis held in Cardiff in 1928 [1] the Pathologist Hubert Turnbull stated "I understand that the primary subject of the discussion is the nature of encephalomyelitis following vaccination. It is no longer necessary to argue that an encephalomyelitis is sometimes a complication of vaccination. The exact cause of the encephalomyelitis need only be discussed."

A plausible explanation for the encephalomyelitis following vaccination which Hubert $M$ Turnbull sought to address is provided by the work of Wakefield et al; who showed Urinary Methylmalonic Acid was significantly raised in all eight children vaccinated with the MMR vaccine who were tested, compared with age-matched controls $(p=0 \cdot 003)$ [2].

Raised urinary methylmalonic acid is a confirmatory test for Vitamin B12 deficiency [3]. A deficiency of Vitamin $\mathrm{B} 12$ is the result of an autoimmune reaction[4] destroying the parietal cells in the stomach thereby inhibiting the uptake of Vitamin B12 in the diet.

"Shaken Baby Syndrome or Vaccine Induced Encephalitis" was the subject of an extensive review by Buttram [5] where he draws attention to the work of others on the subject of demyelination of the central nervous system following the administration of vaccines.

The suggestion by Yazbak [6] that an autoimmune reaction was the basis of adverse vaccine reactions anticipated subsequent observationsand proved to be correct. He stated, "resuming routine vaccination of the newborn period with a Thiomerosal - free vaccine will not be prudent, as the reported reactions seem auto-immune, and do not suggest a heavy metal poisoning".

Yazbak's suggestion of an autoimmune reaction and that of Herroelen, de Keyser and Ebinger [7] who reported 2 patients had neurological symptoms and signs, with evidence of central-nervous-system demyelination, 6 weeks after administration of recombinant hepatitis B vaccine suggesting the time interval would fit a proposed immunological mechanism is further explored here.

The late Dr Archie Kalokerinos was the first to point out that Tissue Scurvy was the cause of what doctors were calling Shaken Baby Syndrome. In his book, Every Second Child, he said, "during one trial the prosecution said scurvy was no longer seen. I replied - "yes it is, but it is not called 'scurvy' it is called Shaken Baby Syndrome"[8]. By reducing or delaying immunization he was able to reduce the mortality in infants.

Tissue Scurvy, unlike the "Seafarer Scurvy" of yesteryear, is a condition in which Vitamin $\mathrm{C}$ is abundant in 
the blood but is unable to enter the cell because of a lack of Insulin which is required to transfer the Vitamin $\mathrm{C}$ into the cell.

Normally insulin binds to its receptor on the cell surface and initiates a chain of events that leads to the insertion in the plasma membrane of a transmembrane glucose transporter called GLUT 4 which facilitates the transport of Vitamin C, glucose and other nutrients into the cell [9]. Within the cell Vitamin $\mathrm{C}$ functions as an antioxidant blocking the damage caused by free radicals, a product of cellular metabolism, and ensures normal cellular function. Tissue Scurvy is the resultof a failure of this process.[10]

Tissue Scurvy involving the liver cells (hepatocytes) leads to under carboxylation of the extrinsic coagulation factors and osteocalcin resulting in bruises, hemorrhages, fractures and encephalopathy [11] - the so-called "triad" of Non-accidental Injury.

The many faces of Tissue Scurvy in Childhood include Sudden Infant Death Syndrome, alleged Non-accidental Injury, Shaken Baby Syndrome, Abusive Head Trauma, Inflicted Brain Injury, Reye's Syndrome, Kawasaki Disease, Anaphylaxis and Diabetes Type 1.[12]

All have one feature in common - hyperglycemia, the signature of Insulin deficiency resulting from vaccines, and other forms of antigenic stress which damage the Beta cells of the Islets of Langerhans resulting inhypoinsulinemia and Tissue Scurvy.

Regrettably doctors in the English speaking world are taught "abused infants may have bleeding around the brain and in the eyes - the hallmarks of SBS but most also bear signs of the violence which killed them, fractures, bruises, burns, malnutrition or neglect"[13].

Adding to the problem is "the American justice system is not designed to determine scientific truth but, rather, to balance contested facts and bring closure to a dispute"[14], and the systems in the UK, Australia, Canada and New Zealand would appear to be similarly afflicted.

It is precisely this failure to seek the truth that is destroying the lives of hundreds, perhaps thousands of innocent familiesand the American justice system and others are urgently in need of change.

Reviewed here are some instances in which a father, family member, or caretaker has been accused of causing the death of an infant or child from Shaken Baby Syndrome while the true cause of death was an autoimmune reaction.

Doctors making these accusations are inadequately trained in the pathophysiological processes of Hemostasis and Osteogenesis which require thecarboxylation of Osteocalcin and some Clotting factors, a process which takes place in the liver and which is essential for the efficient clotting of blood and the prevention of fractures.

Sometimes blood tests are done showing defects in Liver Function but the results are completely ignored or their significance misunderstood and the unfortunate parent bears the consequences of the doctor's ignorance.

\section{Case Report 1}

John, not his real name, was the second child of a 34 year old mother who suffered from severe morning sickness for about 5 months of her pregnancy which lasted 9 months. A forceps delivery was necessary and the infant weighed 3860 gm. There was some bruising on the left side of the face possibly from the forceps. Apgar scores were 8 at 9 and 1 at 5 minutes. Vitamin $\mathrm{K}$ was given by intramuscular injection.

The infant was both breast and formula fed for 3 weeks and then given formula only. He was circumcised and did not bleed excessively but the wound became infected and he was given a course of antibiotics which controlled the infection.

When he was 6 weeks old he was noticed to be very pale and some bleeding occurred from the nose and he developed diarrhea and was "grizzly". Shortly thereafter he had difficulty in breathing and was taken to the nearest hospital.

\subsection{Investigations in Hospital}

1. CT scan showed a Right Sided Subdural Hemorrhage. No retinal hemorrhages were seen

2. Skeletal X-Ray showed Periosteal reaction along the distal half of the right femur and a healing corner metaphyseal fracture of the distal right femur.

3. Ophthalmoscopy showed
a. Right retina clear
b. Left retina "Boat shaped" hemorrhages
involving all layers.

4. Blood Tests

$\begin{array}{lll}\text { 1. Hemoglobin } & 63 \mathrm{gm} / \mathrm{L} & \text { NR } 95-100 \\ \text { 2. Platelets } & 706 \times 10^{9} / \mathrm{L} & 160-600 \\ \text { 3. Monocytes } & 1.3 \times 10^{9} / \mathrm{L} & 0.2-1.2 \\ \text { Blood smear shows } & \end{array}$

a. Fragmented cells

b. Spherocytes

c. Anisocytosis

d. Abnormal monocytes
5. CRP
$13.9 \mathrm{mg} / \mathrm{L} \quad \mathrm{NR}<6.0$
6.Plasma Glucose
$6.1 \mathrm{mmol} / \mathrm{L} \quad 3.0-5.5$

The child failed to respond to treatment and died shortly after admission.

Diagnosis- Non-accidental Injury.

\section{Case 2}

Birth and Early History.

Birth was by normal delivery after a gestation period of 9 months. Apgar scores were 9/5 and 9/10 and screening for T4, PKU AND Galactosemia were all normal. He was given an IM injection of Vitamin K and Hepatitis B Vaccine was given 2 days later. He was breast fed.

Mother noticed this child was different from her two previous children in that "his body and head were never quite in proportion" in that his head was disproportionately large - a known feature of Congenital Toxoplasmosis.

The mother had difficulty in retaining the prenatal 
Vitamins she was prescribed because of morning sickness which lasted the entire nine months of her pregnancy. Significantly during this period there were 5 pet cats in the house.

The presence of cats is a risk factor for the development of Congenital Toxoplasmosis. Babies born with the illness can have consequences such as blindness, hydrocephalus, jaundice, eye lesions, and neurologicalproblems.

He was vaccinated with $\mathrm{DPaT}, \mathrm{HiB}$, and Polio vaccine.

At the age of 10 weeks he suddenly became lethargic, began twitching and passed urine which was tinged with blood and was immediately admitted to hospital..

\subsection{Investigations}

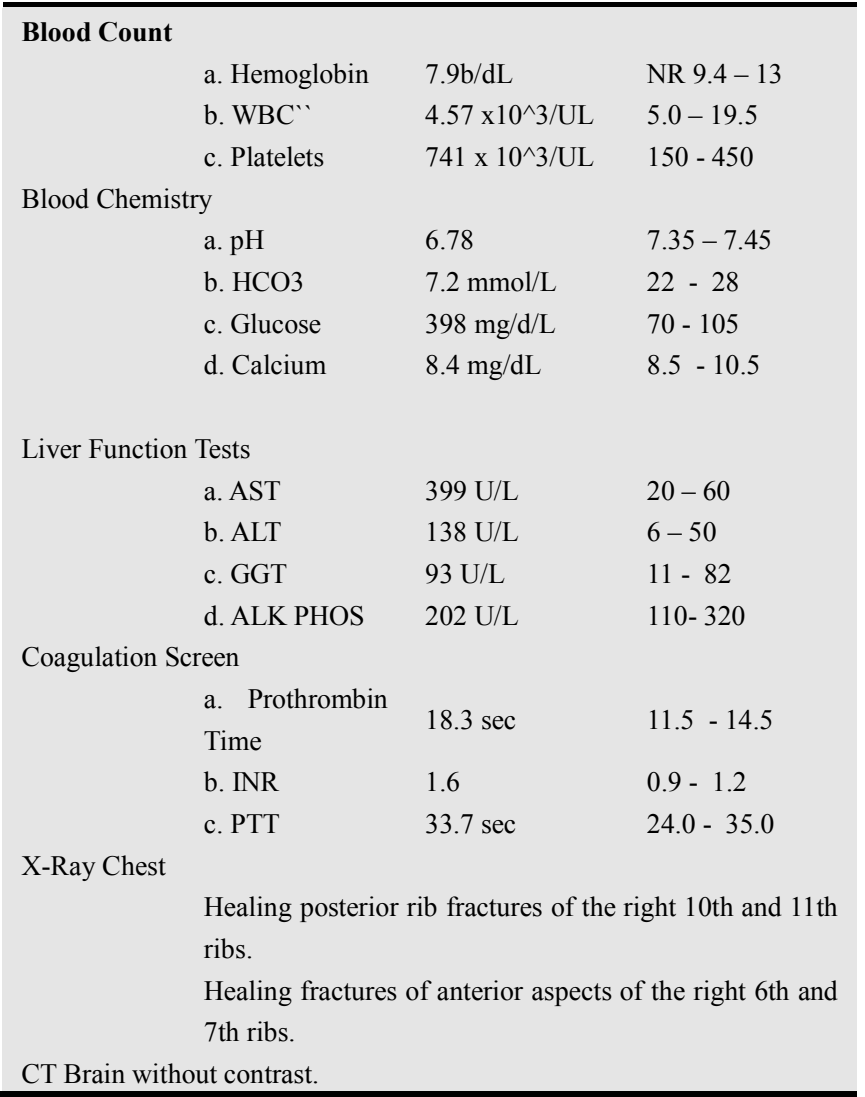

Extensive subdural of mixed density overlying the right greater than the left cerebral hemispheres.

The Diagnosis was Non-accidental Injury and death occurred shortly after admission.

Autopsy confirmed the clinical findings and was reported as a Non-accidental Death.

\section{Case 3}

A $2.9 \mathrm{~kg}$ female infant was born by normal vaginal delivery after a gestation period of 9 months and mother and daughter returned home within 8 hours of the child's birth. Mother noticed a bruise on the child's leg but thought it was accidently bumped by a sibling.

At the age of 8 weeks the child was immunized with DPaT, HiB, Polio and Meningitis vaccine. Within 24 hours of having these vaccines the infant started vomiting intermittently and developed diarrhea and was admitted to hospital.

Appropriate therapy was administered and the infant was fit to return home after a stay of 3 days in hospital.

While at home and being fed by the father she suddenly collapsed, stopped breathing and went limp. An ambulance was called and the child was returned to hospital.

\subsection{Clinical Examination}

The infant was unconscious and numerous bruises were noted on the face and trunk. She was intubated and further investigations were carried out.

\subsection{Investigations}

Skeletal X-Rayexamination was performed which revealed multiple fractured ribs on both sides of the body

\begin{tabular}{lll}
\hline Blood Biochemistry & & \\
\hline 1. Urea & $3.8 \mathrm{mmol} / \mathrm{L}$ & $2.5-7,5$ \\
2. Creatinine & $32 \mathrm{umol} / \mathrm{L}$ & $0-61$ \\
3. Sodium & $142 \mathrm{mmol} / \mathrm{L}$ & $135-146$ \\
4. Potassium & $3.3 \mathrm{mmol} / \mathrm{L}$ & $3.5-5.5$ \\
5. Calcium & $2.32 \mathrm{mmol} / \mathrm{L}$ & $2.37-2.74$ \\
6. Albumin & $28 \mathrm{~g} / \mathrm{L}$ & $35-55$ \\
7. Alkaline Phosphatase & $321 \mathrm{U} / \mathrm{L}$ & $65-265$ \\
8. Alanine Transaminase & $59 \mathrm{U} / \mathrm{L}$ & $5-45$ \\
& & \\
Urine & & \\
1. Protein & Trace & \\
2. Glucose & + by Multistix & \\
\hline
\end{tabular}

The child died shortly after admission and an Autopsy was performed and the cause of death was reported as HEAD INJURY.

The father was arrested and charged with Murder.

\section{Discussion}

In case 1 the blood test showing markedly reduced Hemoglobin with an increase in monocytes, spherocytes, fragmented cells and anisocytosis when associated with an increase in CRP is highly suggestive of a Congenital Mononucleosis, a viral infection, causing an autoimmune hemolytic anemia.

When he was 6 weeks old he had diarrhea, was irritable and was bleeding from the nose. These are allclassical signs that should have alerted one to the possibility of Infantile Scurvy.

The event that necessitated admission to Hospital was the sudden onset of difficulty in breathing which could be interpreted as an Apparent Life Threatening Event (ALTE). The causes of an ALTE are numerous but with evidence of a bleeding disorder causing a neurological lesion would be the most likely cause.

Laboratory investigations demonstrate Liver dysfunction as shown by an abnormal Alanine Transaminase levels and 
the presence of Glucose in the urine confirms hyperglycemia was a feature of the infant's illness

Hoffman and Franck,[15] reported a case of Infectious Mononucleosis, Autoimmunity, and Vasculitisin 1979 and they suggested there is evidence that many of the clinical features of mononucleosis are mediated by the effects of immune complexes on serum and the various tissues in which they are deposited. Mixed cryoglobulins, cold agglutinins, antinuclear antibodies, and rheumatoid factors have been demonstrated in more than half of the cases surveyed for these abnormalities.

This case confirms the results of Hoffman and Frank in that Autoimmunity and Vasculitis are both present. Although neither the Monospot Test nor tests for the Epstein Barr Virus were done the results of the blood test are typical of Infectious Mononucleosis.

In case 2 the child has clear evidence of both defective liver function as shown by the abnormal Liver Function Tests and of hypoinsulinism as demonstrated by the increased level of Glucose in the blood.

The presence of cats is a risk factor for the development of Congenital Toxoplasmosis. The parasite infects the mother and is transferred through the placenta to the foetus. Babies born with the illness can have consequences such as blindness, hydrocephalus, jaundice, eye lesions, and neurological problems and it is probable that it was this parasitic infection which initiated the autoimmune reaction.

In case 3 an abnormal Alanine Transaminase is evidence of a defective Liver function and the presence of Glucose in the Urine establishes hypoinsulinemia. Symptoms started within 24 hours of being vaccinated with 4 different vaccines and it is highly probable that this was indeed an adverse vaccine reaction.

\section{Conclusion}

In all three cases there is clear evidence that an autoimmune response to an antigen, virus, parasite or vaccine caused hypoinsulinism which led to Tissue Scurvy and Tissue Scurvy is frequently misdiagnosed as Shaken Baby Syndrome, Abusive Head Trauma, Inflicted Brain Injury or Non-accidental Injury. The late Dr Mark Struthers reminds us that infantile scurvy, brought to our attention byDr Archie Kalokerinos, is a potential cause of false allegations of child abuse.

A new scientific truth does not triumph by convincing its opponents and making them see the light, but rather because its opponents eventually die, and a new generation grows up that is familiar with it. (T.S. Kuhn, the Structure of Scientific Revolutions).

"All truth passes through three stages. First, it is ridiculed, second it is violently opposed, and third, it is accepted as self-evident". Arthur Schopenhauer

\section{Acknowledgements}

I wish to thank the parents of the children reported here for providing me with the evidence needed to prepare the respective reports and I wish to thank my wife for helpful insights.

\section{References}

[1] Turnbull HM. Encephalo-myelitis In Viral Diseases And Exantemata. Br Med J.1928 August 25,2(3529):331-334

[2] A J Wakefield, SH Murch, A Anthony, J Linnell, D M Casson, et al;RETRACTED:Ileal-lymphoid-nodular hyperplasia, non-specific colitis, and pervasive developmental disorder in children Lancet Vol 351, Number 910328 February 1998

[3] Kwok T, Cheng G, Lai WK, Poon P, Woo J, Pang CP.Use of fasting urinary methylmalonic acid to screen for metabolic vitamin B12 deficiency in older persons.Nutrition. 2004 Sep;20(9):764-8.

[4] Innis MD.Autoimmunity and non-Accidental Injury in ChildrenClinical Medicine Research.MM-DD-2013, pp. xx.doi: $10.11648 / \mathrm{j}$.

[5] Buttram H E. Shaken Baby Syndrome or Vaccine Induced Encephalitis Medical Sentinel;2001:6(3),83-89

[6] Yazbak FE Testimony of Michael Belkin before the Advisory Committee on Immunization Practices - Centers for Disease Control and Prevention February 17,1999 _Atlanta Georgia

[7] Herroelen L, de Keyser J, Ebinger G. Central-nervoussystem demyelination after immunization with recombinant hepatitis B vaccine. Lancet 1991;338:1174-1175.Lancet. 1991 Nov 338(8776):1174-5.

[8] Kalokerinos A. Every Second Child. 1981 pp 3-165 Thomas Nelson (Australia)

[9] Cunningham JJ. The Glucose/Insulin System and Vitamin C:Implications in Insulin-dependent Diabetes Mellitus. J Am CollNutr; 1998:vol 17 p105-108

[10] Cunningham JJ, Ellis SL, McVeigh KL, Levine RE, ,Jorge Calies Escandon Reduced mononuclear leucocyte ascorbic acid content in adults with insulin-dependent diabetes mellitus consuming adequate dietary vitamin C Metabolism 1981; vol 40;148-149.

[11] Innis MD. "Vitamin K Deficiency Disease". Jour of Orthomol Med 2008; vol 23; 15-20

[12] Innis MD. We should harness the power of our colleagues' fresh ideas. BMJ Rapid Responses $24^{\text {th }}$ July 2013.

[13] Squier W. Beyond reasonable doubt Guardian March 13 2008.

[14] Christian CW, Block R, and the Committee on Child Abuse and Neglect Abusive Head Trauma in Infants

[15] Gary S. Hoffman, MD; Walter A. Franck, MD Infectious Mononucleosis, Autoimmunity, and Vasculitis A Case ReportJAMA.1979;241(25):2735-2736 